\title{
Research on Intelligent Material Handling Control System Based on Single Chip Microcomputer
}

\author{
ZHU Chun-yan, DONG Xiao-wei, JIN zhong-bo \\ College of Electrical and Information, Heilongjiang Bayi Agricultural University, \\ Daqing 163319, China \\ 296870704@qq.com
}

\begin{abstract}
The purpose of this system is to carry the car automatically. The AT89S51 single-chip computer is used as the control core. The infrared sensor is used to detect the road and send the signals to the single-chip computer. The car can drive along the fixed track automatically. After reaching the required location, the inductive proximity switch is used to detect the iron sheet. The electromagnet is used to realize the function of automatic fixed-point pick-up and unload, and the counting and timing is carried out automatically. ${ }^{1}$
\end{abstract}

Keywords: AT89S51 Single Chip Microcomputer, Trolley, Intelligence, carry

\section{Introduction}

The system consists of hardware and software. AT89S51 is the core component of the whole car. It controls all the running states of the car[1]. It is responsible for detecting the state of sensors and controlling the motor. The schematic diagram of the system is shown in Figure 1.

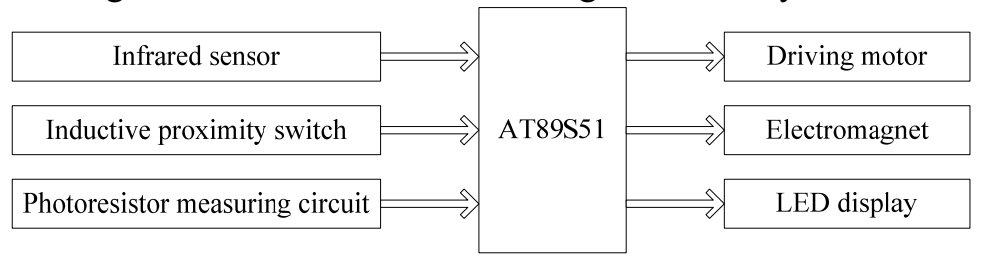

Figure 1. Schematic Block Diagram

The trajectory of the car, as shown in Figure 2, is the principle diagram of the car handling. The trajectory of the car made of black belt is placed at three points A, B and C with small lights respectively. The three points $\mathrm{A}, \mathrm{B}$ and $\mathrm{C}$ are determined by the light source of photosensitive resistance detection[2]. According to the requirements, point $\mathrm{A}$ is the starting point, and the goods to be transported by the car are also placed at point $\mathrm{A}$. When the car reaches

\footnotetext{
${ }^{1}$ Article history:

Received (April 8, 2019), Review Result (May 10, 2019), Accepted (June 6, 2019)

Author's Name: ZHU Chun-yan

Author's profile: ZHU Chun-yan (1978-), Heilongjiang Province,Hegang. Lecturer. Master.

Email:296870704@qq.com.Telphone:13836962218

Correspondent Author: DONG Xiao-wei (1979-), Heilongjiang Province, baiquan. associate professor. doctor. Master's Supervisor.
} 
point $\mathrm{B}$, it needs to unload the cargo and drive back to point $\mathrm{A}$. When there is no goods available at point $\mathrm{A}$, the car stops after driving to point $\mathrm{C}$.

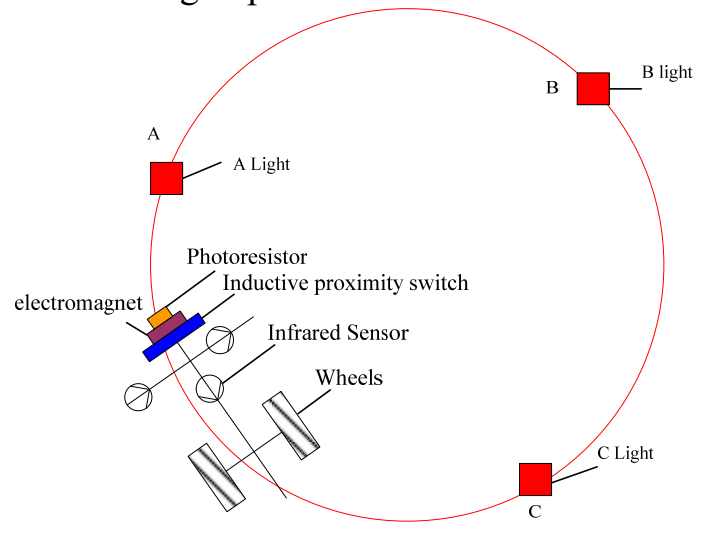

Figure 2. Car handling schematic diagram

\section{Hardware Design}

In order to realize the function of automatic tracing and fetching of the car, each part of this paper is implemented independently. The circuit schematic design is described in detail below[3].

\subsection{Design of Sensor and Conditioning Circuit}

According to the pin function of the sensor, the circuit for detecting black belt is designed, as shown in Figure 3.

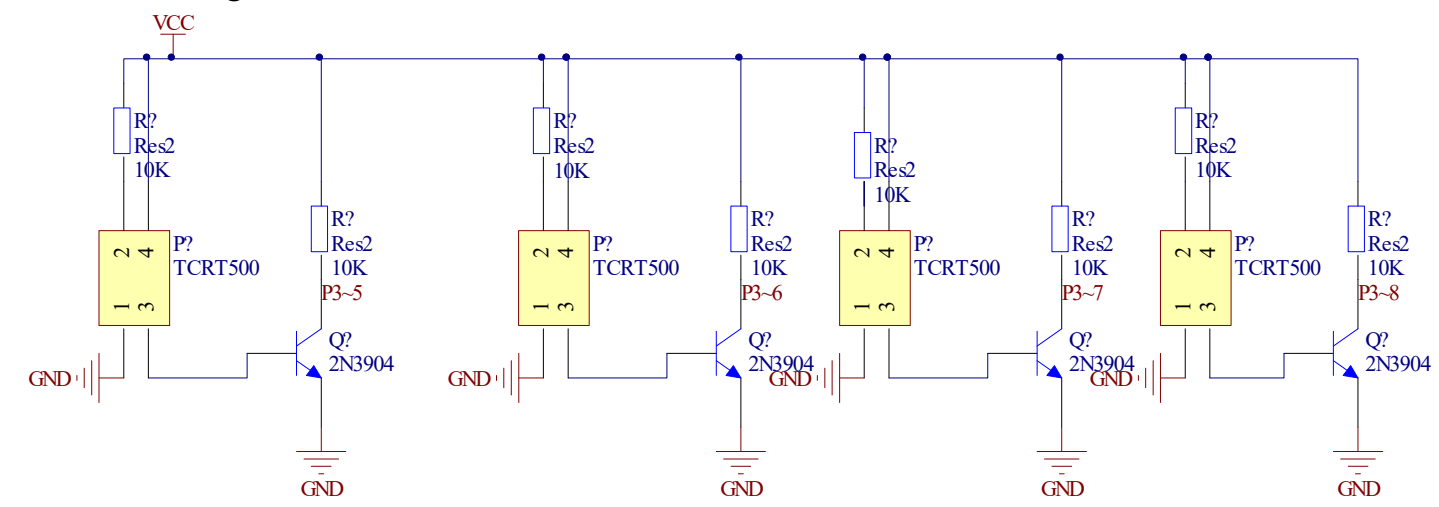

Figure 3. Trace Circuit Diagram

When the sensor works normally, the output level is high. When the sensor can not receive the reflected signal, it will output low level. At this time, the low level signal will be transmitted to AT89S51 single chip computer through P3.5 or P3.7 for processing, so as to determine which pre-programmed program to execute to control the driving state of the car[4]. The installation position of the infrared sensor is shown in Figure 4. 


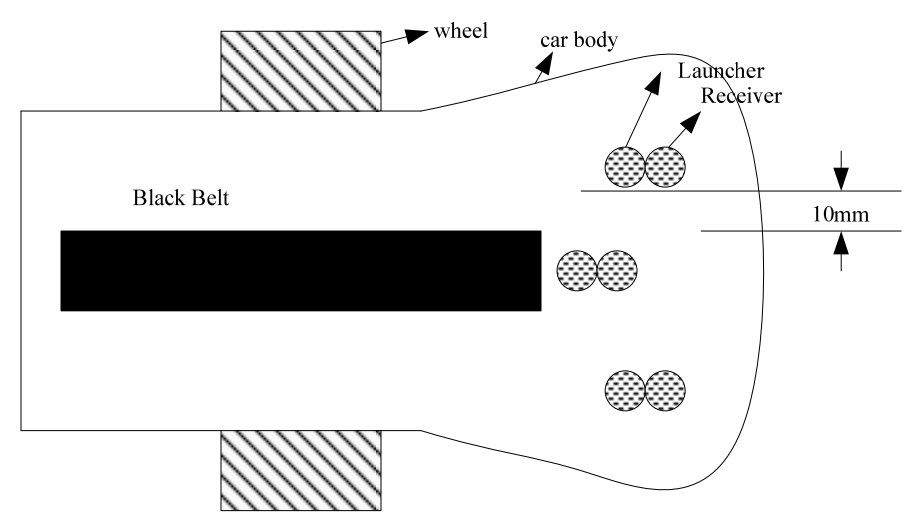

Figure 4. Sensor Installation Location

\subsection{Design of a Circuit for Determining the Operating Position of a Car}

The three points of A, B and C are determined by photoresistors, which are accomplished by photoresistors and $\mathrm{A} / \mathrm{D}$ in the microcontroller[5]. When the photoresistor receives light of different intensity, its resistance value will change. Because of the change of the resistance value of photoresistor, the voltage input to the singlechip P3.3 will change with the change, and the value converted by A/D inside the singlechip will also change. The direction of searching light will be determined by the processing of the value, so as to determine the operation position[6]. The schematic diagram is shown in Figure 5.

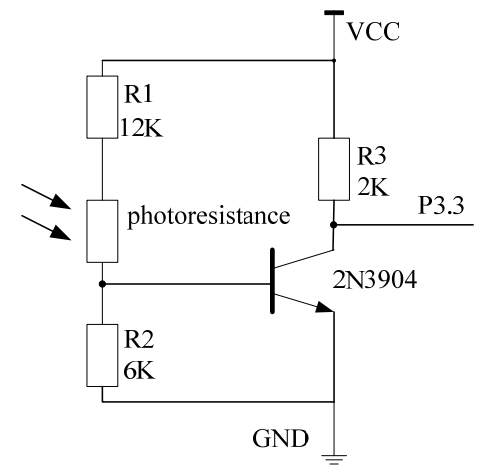

Figure 5. Light-seeking schematic diagram

\subsection{Design of Motor Driving Circuit}

The driving circuit can make the motor rotate. L298N's 5, 7, 10 and 12 pins are connected to the single-chip computer[7]. Through the programming of the single-chip computer, the functions of PWM speed regulation and positive and negative inversion of two DC motors can be realized. That is, AT89S51 outputs two sets of PWM waves, each set of PWM waves is used to control the speed of a motor. The other two I/O ports can control the forward and reverse of the motor. The control method and the control circuit are relatively simple. That is, P1.5 and P1.4 control the direction of the first motor, input: PWML control the speed of the first motor; P1.3 and P1.2 control the direction of the second motor, input PWM2 control the speed of the second motor. The motor chosen here is $3 \mathrm{~V}$ DC motor. Figure 6 is the driving circuit diagram of the motor. 


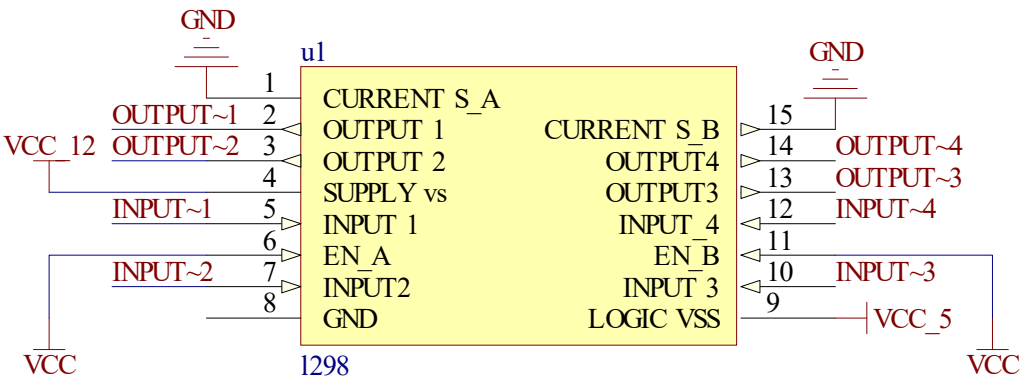

Figure 6. Driver Circuit

When two motors are running at the same speed during the operation of the car, the car will perform direct work. When it is necessary to turn, it is possible to stop or decelerate one motor while the other motor rotates normally. That is to say, when the car needs to turn left, the motor on the left stops turning or decelerates, while the motor on the right runs normally. When turning right, the right motor stops and the left motor runs normally.

\subsection{Circuit Design of Iron Sheet Detection and Material Collection}

The inductance proximity switch LJ12A34Z/BX is adopted. The inductance proximity switch has three interfaces: grounding, power supply and output. Its effective distance measurement is $4 \mathrm{~mm}$, which can meet the design requirements of the car[8]. The inductive proximity switch LJ12A34Z/BX is combined with an electromagnet to complete the detection and handling of iron sheets. The electromagnet is connected by a triode (as a control switch), and the on-off and off of the electromagnet are controlled by the output signal of an I/O chip P3.1 of AT89S51. The schematic diagram is shown in Figure 7.
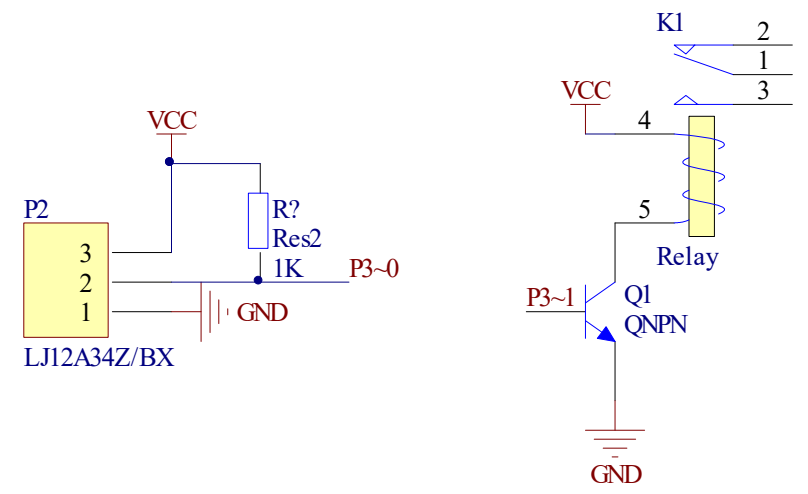

Figure 7. Principle of iron sheet detection and extraction

\subsection{Design of Display Circuit}

Use LED digital tube to display time and number[9]. During the working process of the car, it is necessary to record the time spent and the number of objects taken by the car. The designed display circuit is shown in Figure 8. 


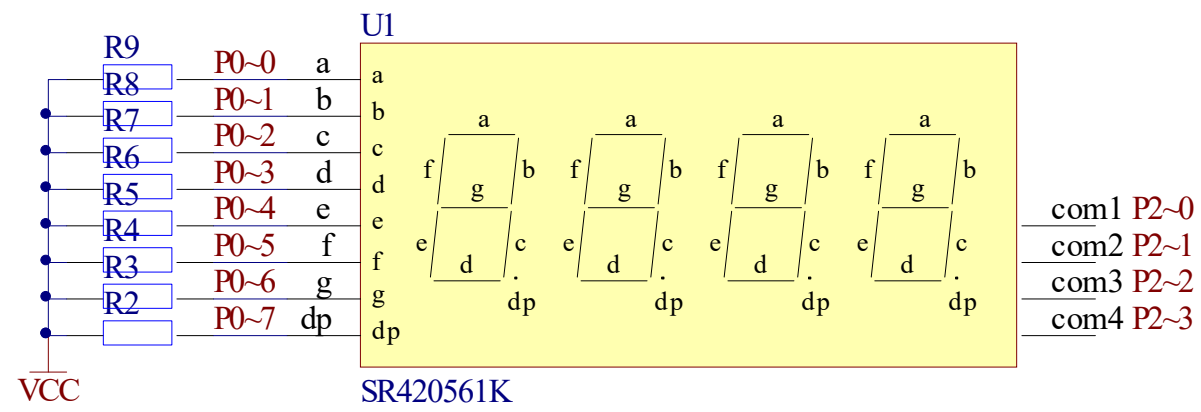

Figure 8. Display Circui

\subsection{Reset circuit and crystal oscillator circuit}

When SCM is initially powered on, it needs reset operation. In the process of operation, the MCU may be disturbed by the outside world, which makes the program fall into dead cycle or "run away". When this happens, it is necessary to reset the MCU in order to restart the operation[10]. The reset circuit and crystal oscillator of MCU are shown in Figure 10.

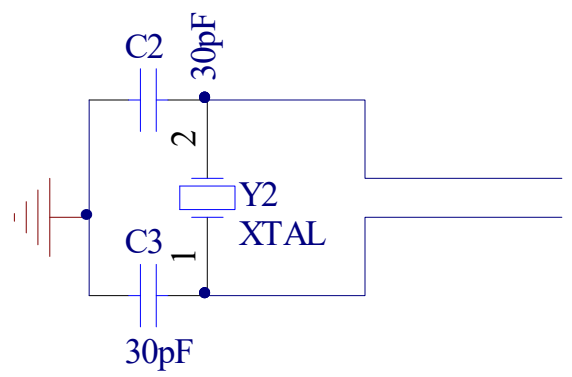

\section{Figure 9. Crystal oscillator and reset circuit}

\section{Software Design}

The flow chart of the main program is shown in Figure 10. From the beginning of the execution of the program, the traceback program is being executed in a continuous cycle. Through the principle of phototaxis, the car is designed to reach the fixed point A, and then the proximity switch is applied to detect whether there is iron sheet. When there is iron sheet, the electromagnet executes the fetching and starts to execute the timing program. When there is no iron sheet, the car stops at point $\mathrm{C}$. When the pick-up is finished, the car continues to drive. When it reaches point $\mathrm{B}$, the iron sheet is lowered. Then the counter adds 1 . Then display timing and counting by LED.

Using modular design, subroutines have track subroutines, fixed-point pick-up subroutines, display subroutines, timing subroutines. 


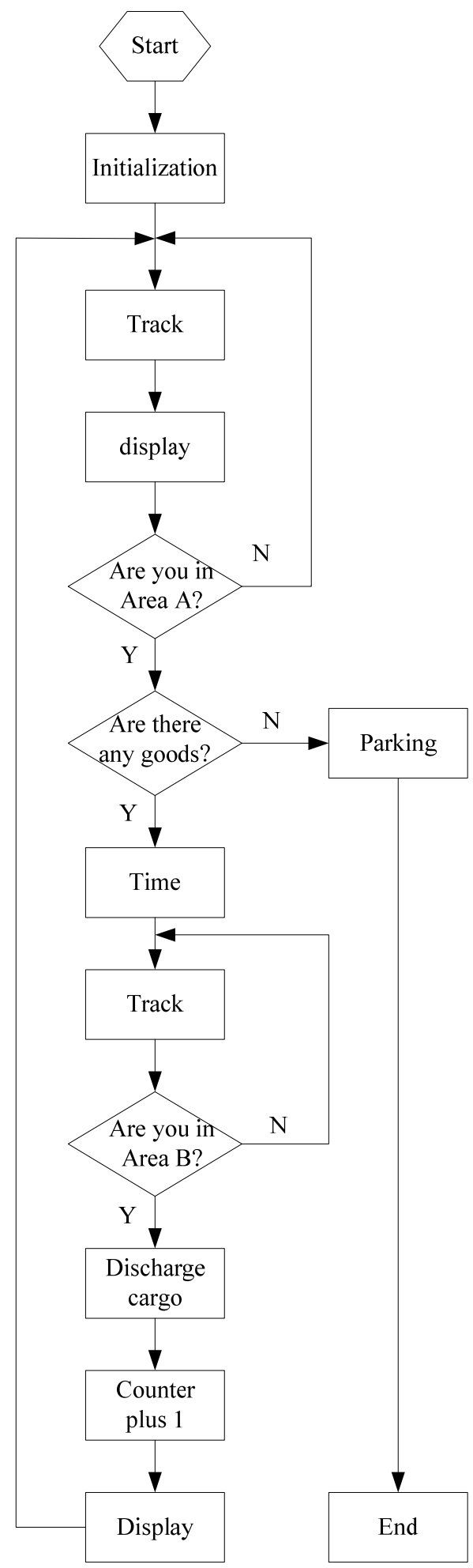

Figure 10. Main program flow chart 


\subsection{Traceback subroutine}

Racing is a task that needs to be done in the whole process of car work. Therefore, in the design, car tracing is the main part of the whole program. The premise of tracing is that the motor rotates to make the car run, and then the photoelectric sensor is used to detect the road and make it run along the fixed track. The flow chart of tracing is shown in Figure 11.

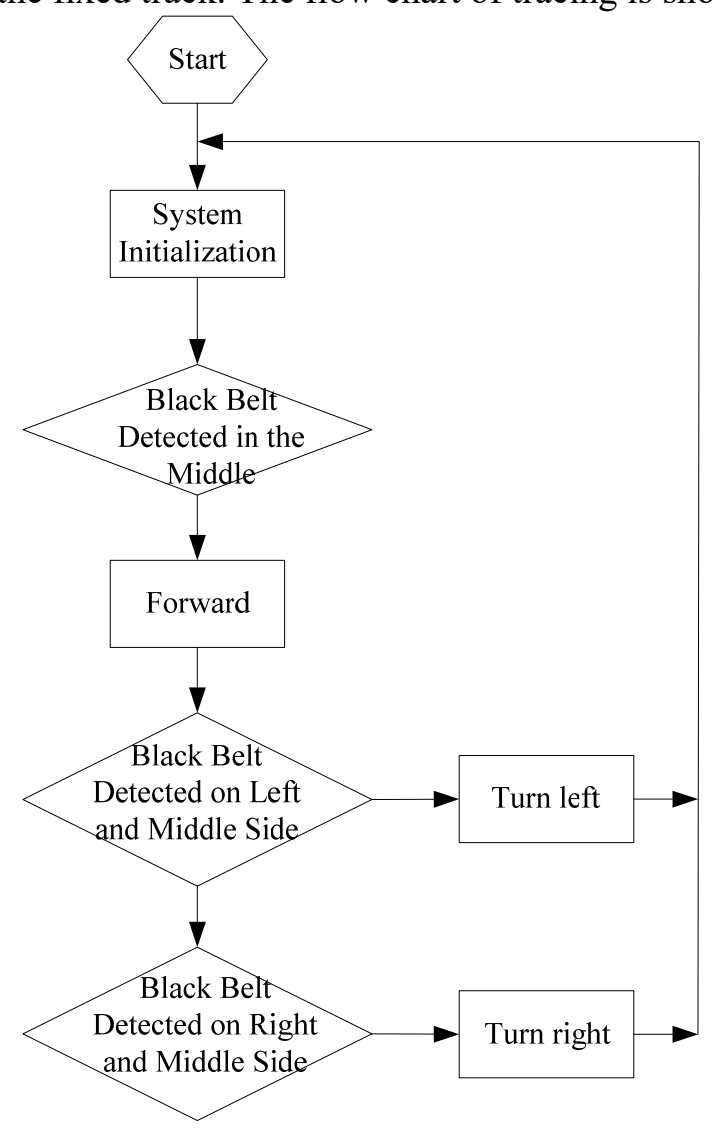

Figure 11. Trace Flow Chart

In the process of tracing, the car first looks at whether the middle sensor detects the black belt or not. If the black belt is not detected, the car carries out direct operation. If the black belt is detected, it is re-detected by program control. When the car deviates from the trajectory, see if the infrared sensor detects the middle and left sensors, and if it detects, turn right. If the sensor detects the right and middle sensors, the car turns left.

\subsection{Location Determination and Object Handling of Cars}

The location of the car is determined in order to enable the car to pick up and unload things at a fixed point. That is to make the car drive to point $\mathrm{A}$ and detect objects at point $\mathrm{A}$, and pick up objects. Then drive the car to point B and place the object at point B. When the car can't detect objects at point $\mathrm{A}$, it should drive to point $\mathrm{C}$ automatically.

The following is a brief introduction to the design of the program through the process, as shown in Figure 12. 


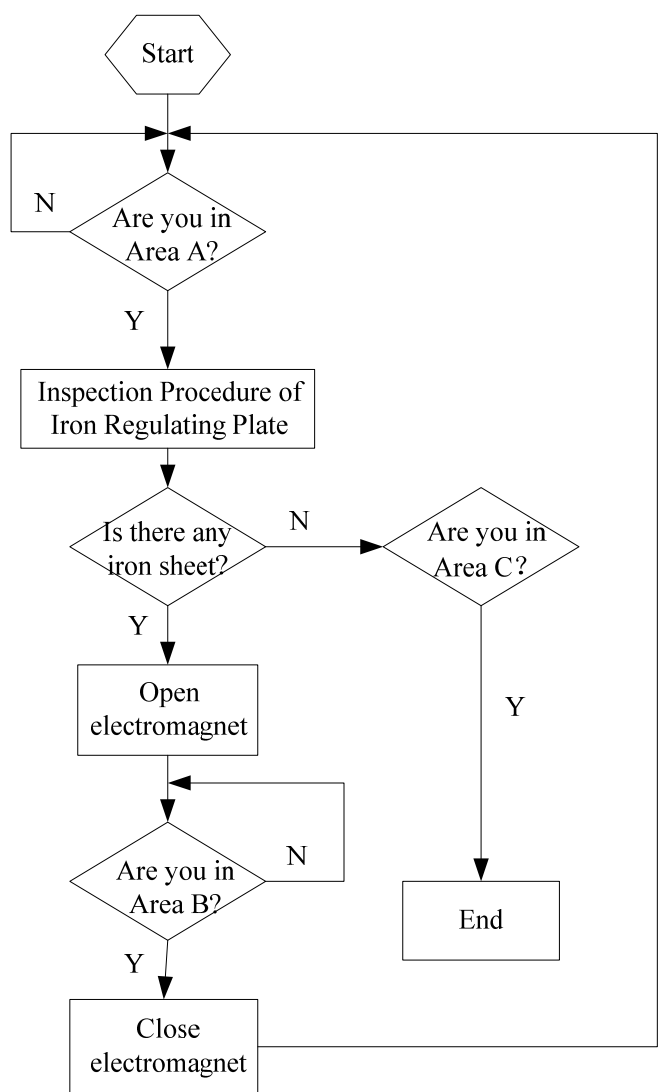

\section{Figure 12. Fixed Point Logistics Flow Chart}

After the car starts driving, when it reaches point A, it detects whether it is point A by photosensitive resistance. If point $A$, it detects whether there is iron sheet. When the iron sheet is turned on, the car continues to run to point B. Similarly, it detects whether it is point B, and if it is point $\mathrm{B}$, it turns off the electromagnet. Then it goes back to point $\mathrm{A}$ and repeats the above operation. When there is no iron sheet at point $\mathrm{A}$, the car tracks to point $\mathrm{C}$, checks whether it is point $\mathrm{C}$, and ends work at point $\mathrm{C}$.

\subsection{Display subroutine}

Through the design of the display circuit, the following display flow chart can be obtained. As shown in Figure 13.

The display of the digital tube starts from the low position. After the position is determined, the segment code is selected by looking up the table and adding delay control. When this light is $\mathrm{P} 2.0=0$, the next bit is selected.

\subsection{Counting and Timing Subprogram}

After the car picks up the object accurately, the timer starts and starts to count. The position of time display is determined by position selection. When the electromagnet is closed, the timer is closed. At this time, the display time is the delivery time of the car. At the same time, the counter is added 1 . When the car returns to point A to pick up, the timer is cleared and the timing is restarted. 


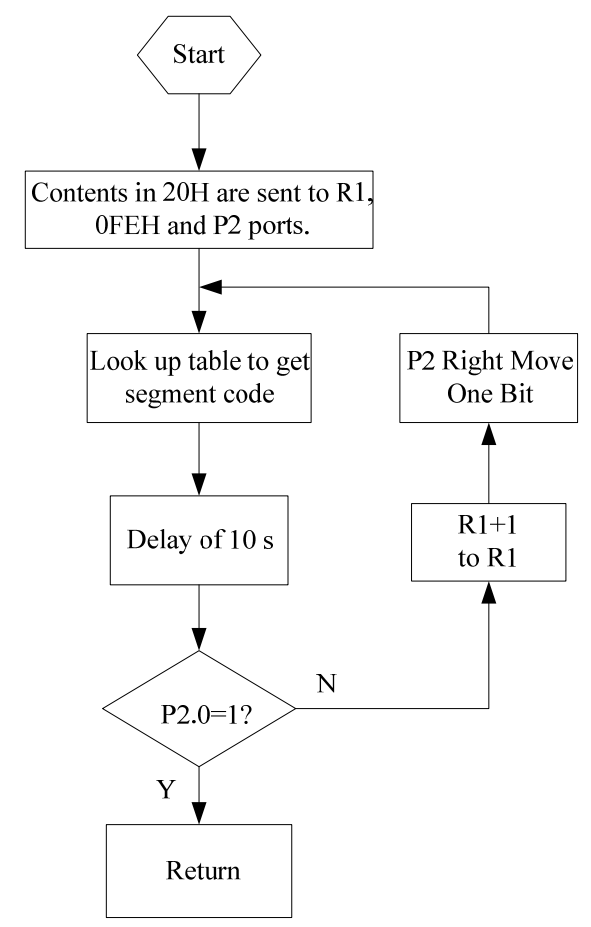

Figure 13. Shows the Flow Chart

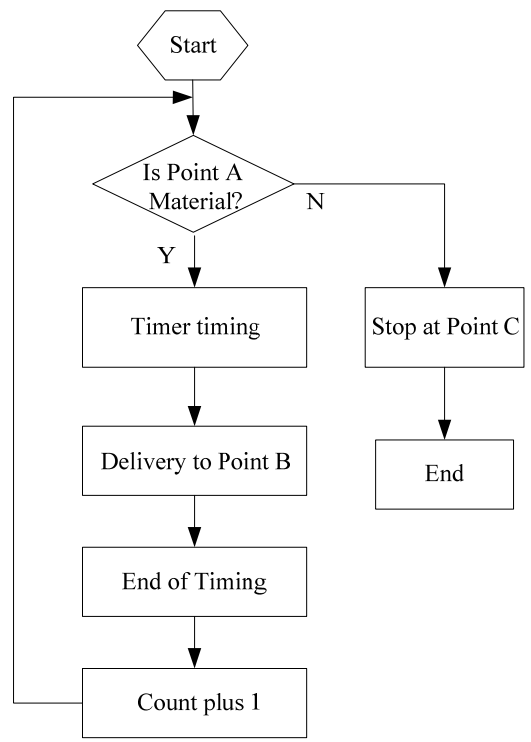

Figure 14. Counting and timing flow chart

\section{Conclusion}

The automobile can realize the function of auto-tracing and traveling according to a certain trajectory, so that the car can start from any point to point $\mathrm{A}$ and reach the requirement of the car to reach a fixed position. Then the goods are detected at point A, picked up automatically, and driven to the target location $\mathrm{B}$ according to the prescribed route before dropping the goods. 
Then the above operations are looped. When the goods can not be detected at point A, the car will automatically drive to point $\mathrm{C}$, where the goods are simulated with iron sheets. No manmade operation is required during the working process of the car. The car needs to travel along a fixed trajectory at all times. When the car deviates from the trajectory, the car can be corrected automatically. When a fault occurs, the operator needs to manually reset the system. At the same time, in the process of work, it is necessary to automatically record the number of handling and the time used.

\section{Acknowledgement}

\section{Project support:}

Higher education teaching reform project in Heilongjiang province(Group no. SJGY20180365 ).

The School Cultivate Project Funding Scheme of Heilongjiang Bayi Agricultural University (Grant no. XZR2017-03)

Planning project for the 13th five-year plan in Heilongjiang province department of education (Group no. GJC1316134).

\section{Reference}

[1] Liu Yixiao. "Development of Electronic Technology and Single Chip Microcomputer". Shenzhou, Vol.3, pp.36 (2014)

[2] Zhang Wei. "Design of Control System for Handling Robot Based on C51 Single Chip Microcomputer", Journal of Zhangzhou Vocational and Technical College, Vol.3, pp.92-94 (2019)

[3] Chen Si. "A self-tracking car tracking scheme optimization system", electronic test, (2017) pp.47-48.

[4] Li Huicong. "Discussion on the method of measuring temperature at more than 20 points in DS18B20". Microcomputer Information, Vol.26, pp.166-167, (2010)

[5] Chang Yuxin, Liman, Hao Gang, Pan Wei. "Hardware Design in Proteus Environment". Modern Industrial Economy and Informatization,Vol.8, No.16, pp.36-37 (2018)

[6] Leopold. "Design and Innovative Application of Minimum SCM System". Scientific and Technological Innovation, Vol.7, pp.26-27 (2019)

[7] Wu Yujun. "Brief analysis of the application and development of single chip computer". Wireless interconnection technology, Vol.13, pp.22-23 (2016)

[8] Zhang Jun. “Intelligent temperature sensor DS18B20 and its application”. Instrument technology, Vol.4, pp.6870 (2010)

[9] Cong Yuhua, Zhu Huijuan, Tian Wenjun and Jin Wenlu. "Hardware design and implementation of a pocket experimental platform based on 51 single chip computer". Digital technology and application, Vol.35, No.12 pp. 153-155 (2018)

[10] He Hong, Zhou Li. "Track Car Control Method and Programming”. Electronic Production, Vol.16, pp. 6-8 (2014) 